L. Schäfer, Qualit. Prüf. neutraler Chininsalze auf Nebenalkaloïde. 1041

in verdünnter Säure auflöst und die saure Lösung durch Uebersättigen mit Ammoniak in Chinin. purum überführt.

Ich werde in einer weiteren Abhandlung nähere Mittheilungen über die quantitative Bestimmung des Cinchonidins im Chininsulfate bringen.

\title{
Die qualitative Prüfung neutraler Chininsalze auf Nebenalkaloïde.
}

\author{
Von Dr. Lou is Sehäfer.
}

Die Oxalatprobe eignet sich in demselben Verhältnisse, wie in dem obigen Aufsatze angegeben, zur Prüfung anderer Chininsalze. Sie kann direct auf diejenigen neutralen Chininsalze angewendet werden, deren Löslichkeit in Wasser nicht geringer ist, als wie die von Chininsulfat, also z. B. auf die Prüfung von Chininhydrochlorat, Bromat, Valerianat. Man verwendet dabei die 1 g kryst. Chininsulfat entsprechende moleculare Menge des betreffenden Chininsalzes zur Probe. Also z. B. $0,90 \mathrm{~g}$ Chininhydrochlorat oder $1,02 \mathrm{~g}$ Bromat, oder $0,97 \mathrm{~g}$ Valerianat and verfährt im übrigen genau wie für Chininsulfat angegeben wurde.

Nebenalkaloïdbeimengungen werden in derselben scharfen Weise angezeigt, wie bei der Probe für Sulfat, doch lässt die Oxalatprobe bei solchen Chininsalzen etwas mehr Nebenalkaloide durch, welche, wie z. B. das baldriansaure Chinin, beim Erhitzen einen Theil ihrer Säure verlieren und alkalisch werden.

Diese directe Anwendbarkeit der Oxalatprobe auf die Prüfung anderer Chininsalze bildet zugleich einen weiteren Vorzug derselben, umsomehr, als bis heute noch keine auch nur annähernd brauchbare Prüfungsmethode für andere Chininsalze bekannt gegeben wurde.

\section{Arbeiten der Pharmakopöe-Commission des deutschen Apotheker - Vereins. \\ Neue Arzeneimittel.}

\section{Antifebrinum. Acetanilid.}

Farblose, glänzende Krystallblättchen ohne Geruch, von schwach brennendem Geschmacke, bei 122 bis $123^{\circ}$ schmelzend, bei $295^{\circ}$ siedend, angezündet ohne Rückstand verbrennend. Sie lösen sich in 